\title{
Combined effect of Chain Extension and Supramolecular Interactions on Rheological and Adhesive Properties of Acrylic Pressure-Sensitive-Adhesives
}

\author{
Xavier Callies ${ }^{a, b^{*}}$, Olivier Herscher ${ }^{c}$, Cécile Fonteneau ${ }^{c}$, Alexis Robert ${ }^{a, b}$, Sandrine Pensec ${ }^{c}$, Laurent \\ Bouteiller, Guylaine Ducouret ${ }^{a, b}$, Costantino Creton ${ }^{a, b^{*}}$ \\ ${ }^{a}$ Laboratoire Sciences et Ingénierie de la Matière Molle, CNRS, ESPCI Paris, PSL Research University, 10 \\ rue Vauquelin, Paris, France \\ ${ }^{b}$ Laboratoire Sciences et Ingénierie de la Matière Molle, Université Pierre et Marie Curie, Sorbonne- \\ Universités, 10 rue Vauquelin, France \\ 'Sorbonne Universités, UPMC Univ Paris 06, CNRS, IPCM, Chimie des Polymères, F-75005 Paris, France \\ xavier.callies@etu.upmc.fr, costantino.creton@espci.fr
}

Key-words: supramolecular chemistry, urea, hydrogen bonds, epoxy, amine, acrylic, tack, network, viscoelasticity

\begin{abstract}
A new approach for the elaboration of low molecular weights pressure-sensitive-adhesives based on supramolecular chemistry is explored. The synthesis of model systems coupled with probe-tack tests and rheological experiments highlights the influence of the transient network formed by supramolecular bonds on the adhesion energy. The first step of our approach consists in synthesizing poly(butylacrylate-co-glycidyl methacrylate) copolymers from a difunctional initiator, able to self-associate by four hydrogen bonds between urea groups. Linear copolymers with a low dispersity $\left(\mathrm{M}_{\mathrm{n}}=10 \mathrm{~kg} / \mathrm{mol}, \mathrm{Ip}<1.4\right)$ have been synthesized via Atom Transfer Radical Polymerization. Films of the copolymers were then partially crosslinked through reaction of the epoxy functions with a diamine. The systematic variation of the average ratio of glycidyl methacrylate and diamine per copolymer shed light on the respective role played by the supramolecular interactions (between bis-urea groups and with the side chains) and by the chains extension and branching induced by the diamine/epoxy reaction. In this strategy, the adhesive performance can be optimized by modifying the strength of stickers (via the structure of the supramolecular initiator, for instance) and the polymer network (e.g. via the length and level of branching of the copolymer chains) in order to approach commercial PSA-like properties (high debonding energy and clean removal).
\end{abstract}




\section{Introduction}

Pressure Sensitive Adhesives ${ }^{1}$ (PSAs) are thin films that stick on almost any surface by simple contact and that can be ideally detached from the substrate without any residue. These materials are used to make ubiquitous objects in our daily life such as adhesive tapes or Postit $^{\circ}$. Their adhesive properties are based upon an optimum balance between their elastic and dissipative properties and are characterized with standard tests of the trade such as tack, peel force and sheer resistance ${ }^{2}$. Like liquids, these materials create easily a molecular contact with the substrate, relax stresses and dissipate energy upon deformation. Like solids, PSAs resists shear forces over long times. Commercial adhesives are often composed of thin viscoelastic polymer films that contain highly entangled and branched polymer chains and the optimization of their rheology requires complex formulation and/or several processing steps $^{3,4}$. The shear resistance is optimal with a light degree of chemical or physical crosslinking while a low molecular weight and polar additive, a "tackifier" is sometimes needed to get a low viscoelastic modulus (below $\sim 0.1 \mathrm{MPa})^{1}$.

As recently pointed out by different authors ${ }^{5,6}$, supramolecular chemistry can be a promising toolbox to elaborate innovative and highly tunable materials. The supramolecular materials are composed of polymer chains functionalized by highly interacting moieties, sometimes called "stickers". The specific association of these stickers via non covalent bonds (usually ionic or hydrogen bonds) induce the formation of a transient network or nanostructure ${ }^{7}$ into the bulk and thus, favors a solid behavior at small deformations. However, at large deformations, the dissociation of stickers can lead to very dissipative properties ${ }^{8}$ and thus, within the framework of soft adhesives, to a high debonding energy ${ }^{9}$. Thanks to their polarity and specific interactions, stickers favor high energy surfaces ${ }^{10,11}$ in dry environment and can improve adhesion properties in severe conditions, such as humid atmosphere or under water ${ }^{12}$.

Among the systems reported in the literature ${ }^{6}$, some of us investigated the self-assembly ${ }^{13}$, rheology ${ }^{14}$ and adhesive properties ${ }^{15}$ of unentangled polymer chains bearing a single tri- or bis-urea sticker per chain located in its middle ${ }^{8}$. The self-assembly of the stickers into nanorods structured in bundles induces a soft elastic behavior at small deformation while a strong strain softening behavior was observed in adhesion tests leading to a localized cohesive fracture of the adhesive layer and relatively low values of adhesion energy. The present study aims at modifying the non linear rheology of these supramolecular structures to better approach PSA-like properties, i.e. high debonding energy and an interfacial failure with a minimum of residue. The specific properties required in a PSA impose to have strainhardening mechanisms in large strain ${ }^{16,17,18}$ which were missing in the previously studied materials. Inspired from insightful studies on the non linear rheology of polymers ${ }^{19,20,21,22}$, our strategy to favor these mechanisms is to increase the degree of branching and entanglements of the supramolecular chains via chain extension and/or cross-linking ${ }^{23,24}$. 
To reach our objective, poly(butylacrylate -co- glycidyl methacrylate) chains centerfunctionalized by a bis-urea sticker were synthesized by Atom Transfer Radical Polymerization (ATRP). As shown in figure 1, the butylacrylate (BA) and glycidyl methacrylate (GMA) monomers were chosen for the project since the BA monomer is widely used as a base polymer for soft adhesives due to its low $\mathrm{T}_{\mathrm{g}}$ and the GMA monomer can easily react with difunctional nucleophilic cross-linkers (or chain extenders). By analogy with classical epoxy resins, a diamine (1,5-amino-2-methylpentane) is thus used to create covalent bonds between the side chains. The cross-linking step occurs in the thin films after the solvent is evaporated.
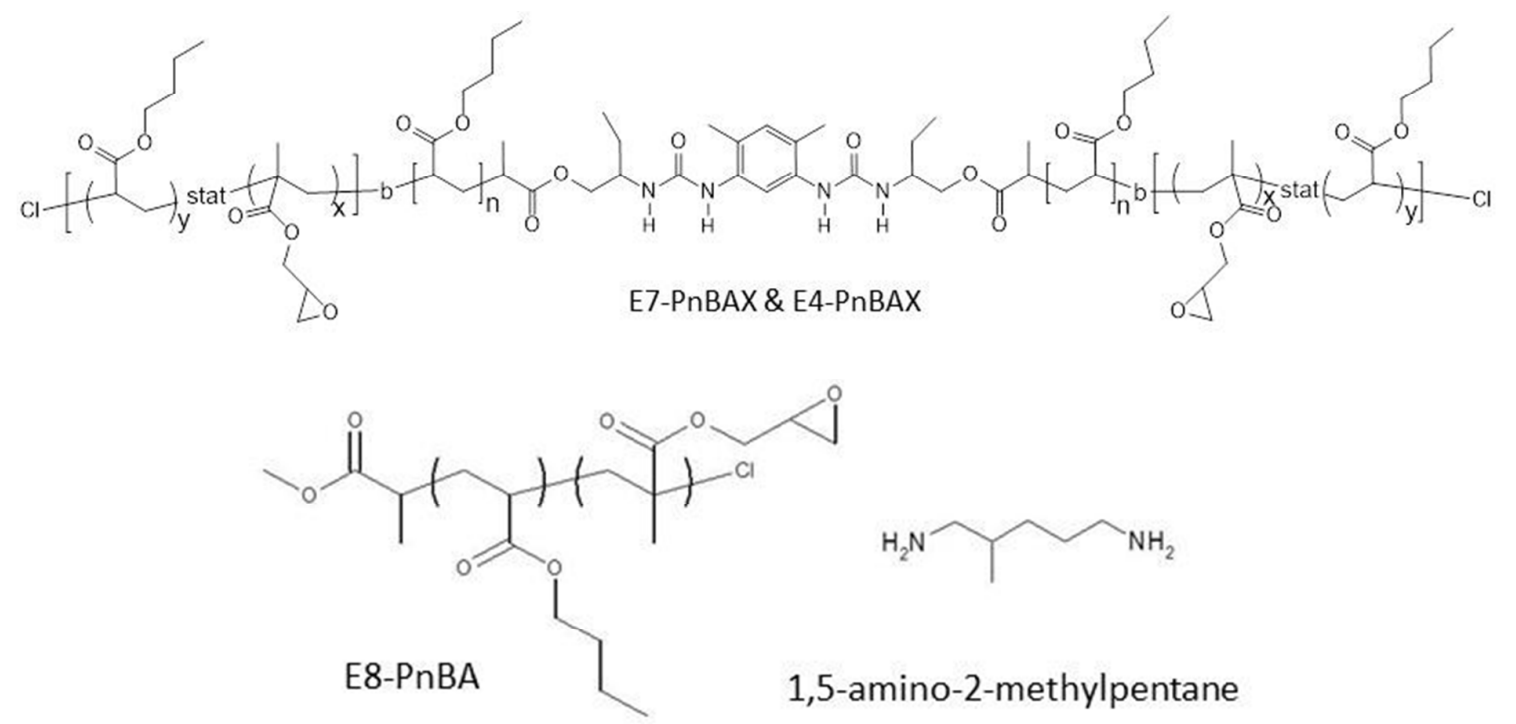

Figure 1: Chemical structure of the copolymers and the diamine used as a cross-linker. For E4-PnBAX, $n=16.5 ; \mathrm{x}=1.75 ; \mathrm{y}=16.5$. For E7-PnBAX, $\mathrm{n}=25.5 ; \mathrm{x}=3.5 ; \mathrm{y}=15$.

Our supramolecular copolymers are centrally functionalized by a bis-urea core able to selfassociate by four hydrogen bonds ${ }^{25}$ between urea groups (see Figure 1). The polar core also plays the role of an initiator ${ }^{26}$ during the polymerization step and two symmetric side chains grow from each side of the sticker. As recently reported, the viscoelasticity ${ }^{27}$ of the bis-urea center-functionalized poly(butylacrylate) (PnBAX) system is mainly regulated by the supramolecular interactions for $\mathrm{M}_{\mathrm{n}}<20 \mathrm{~kg} / \mathrm{mol}$ while it is mainly governed by the relaxation of the PnBA side chains for higher $\mathrm{M}_{\mathrm{n}}$. As the central objective of our study is to focus on the high potential of the supramolecular chemistry for adhesives, the molecular weight of the copolymers was set at $\mathrm{M}_{\mathrm{n}} \sim 10 \mathrm{~kg} / \mathrm{mol}$. Two concentrations of epoxy groups per chain are investigated. E7-PnBAX is a copolymer with an average number of 7.1 epoxy groups per chain while E4-PnBAX contains on average 3.5 epoxy groups per chain (see table 1).

After a brief review of the synthesis of our materials, the linear rheological and adhesive properties of the supramolecular copolymers will be presented. In order to observe the effect of the supramolecular interactions, E4-PnBAX and E7-PnBAX will be compared to two linear bis-urea center-functionalized poly(butylacrylate) polymers (PnBAX5 and PnBAX8) and to a random poly(butylacrylate - glycidyl methacrylate) copolymer (E8-PnBA) synthesized from a 
commercial initiator, which is not expected to interact by strong hydrogen bonds. The incorporation of E8-PnBA in the study aims at detecting possible effects of hard segments introduced by glycidyl methacrylate monomer sequences relative to a bisurea functionalized PnBA of equivalent molecular weight. In a second part, the characterization of supramolecular resins including both the glycidyl copolymers and the diamine will be shown and compared with E8-PnBA-based resins.

\section{Experimental Section}

\section{Materials}

The polymers were synthesized by ATRP via a functional initiator approach, as reported by Fonteneau et $\mathrm{al}^{26}$. Bis-urea center-functionalized polymers (PnBAX5 and PnBAX8) and copolymers (E7-PnBAX and E4-PnBAX) were synthesized from a bis-urea initiator ${ }^{26}$ while the E8-PnBA copolymer was synthesized from a commercial initiator (2-methylbromopropionate). Since the glycidyl methacrylate monomer is more reactive than the butylacrylate monomer ${ }^{28}$, the polymerization of the copolymers was carried out in two steps. n-butylacrylate was polymerized first up to a given conversion level and then a mixture of glycidyl methacrylate and n-butylacrylate was added to the reactor (see SI). Therefore, glycidyl methacrylate monomers are expected to be positioned preferentially towards the middle of the side chains. For all copolymerizations, for both the commercial initiator and the bis-urea initiator, the chromatograms show a displacement of the peak toward higher molar masses with increasing polymerization time (see figure S1). As shown in table 1, the control of the polymerizations was confirmed by the low dispersity measured by Size Exclusion Chromatography (SEC). The average number of glycidyl methacrylate per chain $\left(\mathrm{DP}_{\mathrm{e}}\right)$ was measured by NMR (see figure S2).

$\begin{array}{ccccc}\text { Material } & \mathbf{M}_{\mathbf{n}}(\mathbf{k g} / \mathbf{m o l}) & \mathbf{D} & \mathbf{D P}_{\mathbf{e}} & \text { Initiator } \\ \text { PnBAX5 } & 5.4 & 1.18 & 0 & \text { bis-urea } \\ \text { PnBAX8 } & 7.5 & 1.23 & 0 & \text { bis-urea } \\ \text { E8-PnBA } & 13.8 & 1.25 & 8 & \text { 2-methyl-bromopropionate } \\ \text { E7-PnBAX } & 11 & 1.37 & 7.1 & \text { bis-urea } \\ \text { E4-PnBAX } & 9.1 & 1.35 & 3.5 & \text { bis-urea }\end{array}$

Table 1: Composition and characteristics of the materials: number average molar mass $\left(M_{n}\right)$, molar mass distribution $(Đ)$, average number of glycidyl methacrylate per copolymer chains $\left(\mathrm{DP}_{\mathrm{e}}\right)$ and chemistry of the polymerization initiator. $\mathrm{M}_{\mathrm{n}}$ and $\mathrm{D}$ are both measured by SEC and $\mathrm{DP}_{\mathrm{e}}$ was measured by NMR (figure S2).

In the study of copolymers cured by diamine, three amine/epoxy molar ratios $\left(A_{m} / E_{p}\right)$ were investigated for each copolymer: 1/100, 5/100 and 20/100 (see table 2). These ratios (that correspond to $2 / 100,10 / 100$ and 40/100 N-H/epoxy ratios) are very low because our objective 
was to slightly increase the average chain length of the copolymer and not to create a dense covalent network. For each ratio $A_{m} / E_{p}$, the concentration of diamine [D] and the diamine/chain ratio $(\mathrm{D} / \mathrm{C})$ were calculated. At a fixed molar ratio $\mathrm{A}_{\mathrm{m}} / \mathrm{E}_{\mathrm{p}}$, these two parameters increase with the average number of glycidyl methacrylate monomers per copolymers, since all materials have a similar molecular weight.

The calculation of the two parameters $\mathrm{D} / \mathrm{C}$ and $[\mathrm{D}]$ is illustrated with the following example of E8-PnBA-D1. This resin is made by curing the E8-PnBA with diamine for a molar ratio of one amine moiety for 100 epoxy moieties. As the average number of epoxy groups per chain is 8 (see table 1) and each cross-linker contains two amine groups, the cross-linker/chain ratio $\mathrm{D} / \mathrm{C}$ is given by:

$$
\frac{\mathrm{D}}{\mathrm{C}}=\frac{1}{2} * 8 * \frac{1}{100}=\frac{4}{100}
$$

And the concentration of the cross-linker $[\mathrm{D}]$ is:

$$
[\mathrm{D}]=4 \mathrm{M}_{\mathrm{w}, \mathrm{D}} /\left(4 \mathrm{M}_{\mathrm{w}, \mathrm{D}}+100 \mathrm{M}_{\mathrm{w}, \mathrm{copo}}\right)
$$

with the molecular weight of the copolymer $\left(\mathrm{M}_{\mathrm{w}, \mathrm{copo}}\right)$ and that of the cross-linker $\mathrm{M}_{\mathrm{w}, \mathrm{D}}$.

$\begin{array}{ccccc}\text { Material } & \text { Copolymer } & \mathbf{A}_{\mathbf{m}} / \mathbf{E}_{\mathbf{p}} & {[\mathbf{D}] \mathbf{( \% )}} & \mathbf{D} / \mathbf{C} \\ \text { E8-PnBA-D1 } & \text { E8-PnBA } & 1 / 100 & 0.04 & 4 / 100 \\ \text { E8-PnBA-D5 } & \text { E8-PnBA } & 5 / 100 & 0.19 & 20 / 100 \\ \text { E8-PnBA-D20 } & \text { E8-PnBA } & 20 / 100 & 0.77 & 80 / 100 \\ \text { E7-PnBAX-D1 } & \text { E7-PnBAX } & 1 / 100 & 0.03 & 3.6 / 100 \\ \text { E7-PnBAX-D5 } & \text { E7-PnBAX } & 5 / 100 & 0.17 & 18 / 100 \\ \text { E7-PnBAX-D20 } & \text { E7-PnBAX } & 20 / 100 & 0.69 & 71 / 100 \\ \text { E4-PnBAX-D1 } & \text { E4-PnBAX } & 1 / 100 & 0.02 & 1.8 / 100 \\ \text { E4-PnBAX-D5 } & \text { E4-PnBAX } & 5 / 100 & 0.10 & 8.8 / 100 \\ \text { E4-PnBAX-D20 } & \text { E4-PnBAX } & 20 / 100 & 0.39 & 35 / 100\end{array}$

Table 2: Chemical composition for cross-linker/copolymers blends: $A_{m} / E_{p}$ is the molar ratio between amine and epoxy groups in the polymer matrix, $[\mathrm{D}] \%$ is the weight percentage of cross-linker in the final mixture relative to the total weight, $\mathrm{D} / \mathrm{C}$ is the cross-linker/chain ratio.

For the characterizations of the copolymers by probe-tack tests, $100 \mu \mathrm{m}$ thick films were prepared. Polymer samples were first dissolved in toluene and then, $2 \mathrm{~mL}$ of the solution was deposited on a glass slide $\left(100 * 26 * 2 \mathrm{~mm}^{3}\right)$. The glass slide was left for 2 days at room temperature, and then for 2 more days at $80^{\circ} \mathrm{C}$ to remove the solvent by evaporation. In the case of supramolecular resins, cross-linkers and copolymers were separately dissolved in toluene and then, solutions were mixed in suitable proportions. Like for copolymers, thin 
adhesive layers of supramolecular resins were prepared from these solutions and chemical cross-linking actually occurred during the evaporation step (see Figure S3-S5). After the probe-tack tests, the thin layers were removed from the glass slides for oscillatory tests in linear rheology.

\section{Linear Rheology}

Dynamic rheological measurements were performed on a strain-controlled rheometer ARES LS1 (TA Instruments). The frequency dependence of the viscoelastic moduli $G$ ' and $G$ ' 'was characterized with a parallel plate geometry (diameter 8 and $25 \mathrm{~mm}$ ) at $25^{\circ} \mathrm{C}$ and $80^{\circ} \mathrm{C}$. The ARES LS1 rheometer was equipped with a Peltier device to regulate the temperature of the sample from 0 to $80^{\circ} \mathrm{C}$. Samples were annealed for 2 hours between the two plates at constant temperature before measurements.

\section{Adhesion}

The home-made probe tack set-up used is built on a MTS tensile machine ${ }^{29}$. The motion of the probe is controlled by a hydraulic device and a semi-transparent mirror positioned at $45^{\circ}$ above the adhesive layer gives a top-view of the debonding process. This allows both a direct observation for the experimentalist and the recording of the debonding process by a camera. The alignment between the adhesive and the probe is adjusted thanks to a tripod system ${ }^{29}$. The probe used in the probe-tack tests are steel cylindrical probes with a mirror-like flat surface (diameter $\varnothing=1.0 \pm 0.1 \mathrm{~cm}$ ).

At the beginning of the adhesion test, the probe comes into contact with the adhesive layer at a constant speed until a given normal force $\left(F_{N}=-70 \mathrm{~N}\right)$ is reached. The probe is stopped and after a set time $\left(t_{c}=10 \mathrm{~s}\right)$, the probe is removed at a constant velocity $\left(V_{d e b}=100 \mu \mathrm{m} / \mathrm{s}\right)$. The force $F(t)$ is measured by a load cell $(2500 \mathrm{~N} \pm 0.5 \mathrm{~N})$ and the displacement of the probe $d(t)$ is measured with a LVDT extensometer (range $10 \mathrm{~mm} \pm 0.5 \mu \mathrm{m}$ ). The displacement $d(t)$ provides a measurement of the thickness $h(t)$ of the adhesive during the debonding test:

$$
h(t)=d(t)-K^{*} F(t)+h_{0}(1)
$$

with $h_{0}$ the initial thickness of the layer and $K$ the compliance of the overall system: $K=0.15$ $\mu m . / N \pm 0.01 \mu \mathrm{m} / N$. Probe-tack curves conventionally plot the nominal stress $\sigma_{0}(t)=F(t) / S_{0}$ versus the nominal strain $\varepsilon_{0}(t)=\left(h(t)-h_{0}\right) / h_{0}$ where $S_{0}$ is the contact area between the probe and the adhesive layer at the maximum compression stage. The area under the $\sigma_{0}=f\left(\varepsilon_{0}\right)$ curve is used to calculate the debonding energy of the soft layer, $W_{a d h}(\mathrm{~J} / \mathrm{m} 2)$, i.e. the energy necessary to detach the probe from the layer for the given traction speed:

$$
W_{a d h}=h_{0} \int \sigma_{0} d \varepsilon_{0}(2)
$$


Results

\section{Center-functionalized Copolymers}

The frequency dependence of the storage and viscous moduli at $25^{\circ} \mathrm{C}$ is shown for the two PnBAX and for the copolymers in figure 2. Like PnBAX8, E8-PnBA behaves as a viscoelastic liquid in that frequency and temperature range: $G$ ' $>G$ ' for all $\omega$. A direct comparison of the low frequency region between PnBAX8 and E8-PnBA shows also that the presence of 8 epoxies per chain does give rise to a significantly lower viscosity than the presence of a single bis-urea moeity. PnBAX5, E7-PnBAX and E4-PnBAX are characterized by a transition between an elastic zone at high frequency and a viscous zone at low frequency. A crossing point between $G$ ' and $G$ ' ' is detected at: $\omega_{c}=0.4 \mathrm{rad} / \mathrm{s}( \pm 0.1)$ for E4-PnBAX, $\omega_{c}$ $=0.16 \mathrm{rad} / \mathrm{s}( \pm 0.05)$ for E7-PnBAX and $\omega_{c}=1.8( \pm 0.3) \mathrm{rad} / \mathrm{s}$ for PnBAX5.
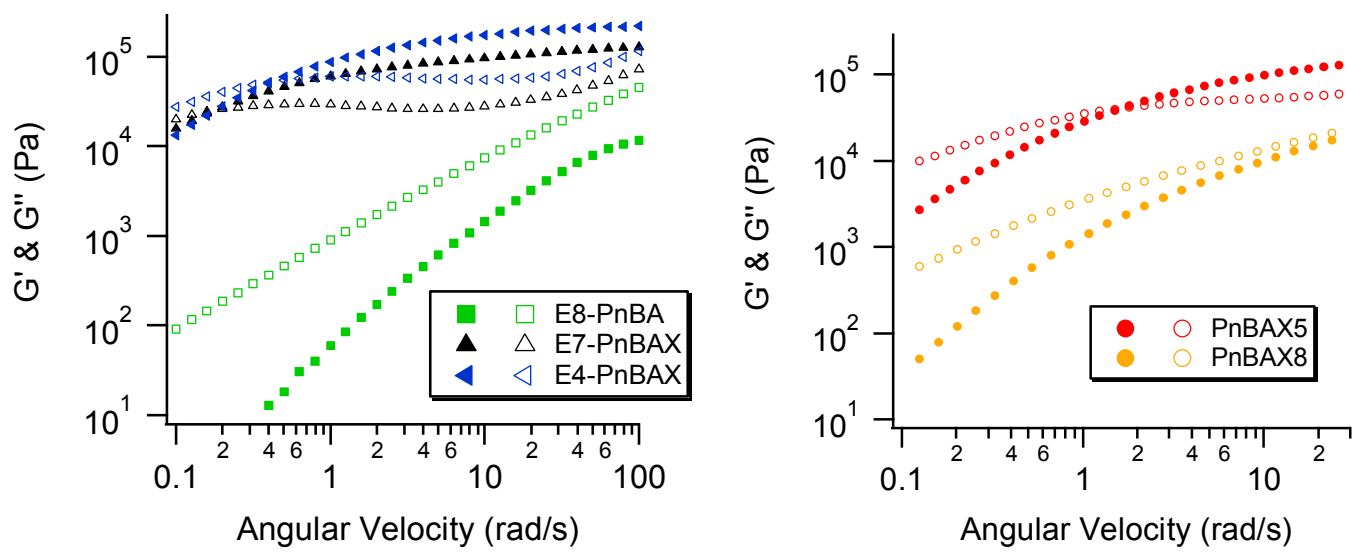

Figure 2: Frequency dependence of $G$ ' and $G$ " in the linear regime at $25^{\circ} \mathrm{C}$ for E8-PnBA, E7-PnBAX, E4-PnBAX, (left), PnBAX5 and PnBAX8 (right). Filled markers represent $G$ ' and unfilled markers, $G^{\prime \prime}$.

In order to probe their adhesive character, the supramolecular polymers were characterized by probe-tack tests with a debonding velocity $V_{d e b}=100 \mu \mathrm{m} / \mathrm{s}$ and at room temperature. It should be pointed out that a proper characterization of pressure-sensitive-adhesive properties would have implied also peel test characterizations and resistance to long term shear. Due to the proof of concept nature of this study only probe tack experiments were carried out. However an estimate of the peel force at equivalent temperatures and debonding rates ${ }^{30,18}$ can be obtained by converting the adhesion energy in $\mathrm{J} / \mathrm{m}^{2}$, in an estimated peel force in $\mathrm{N} / \mathrm{m}$. Therefore an adhesion energy of $100 \mathrm{~J} / \mathrm{m}^{2}$ in tack corresponds roughly to a peel force of 1 $\mathrm{N} / \mathrm{cm}$. 
For all copolymers, the debonding process was cohesive, i.e. macroscopic residues were observed on the steel probe after the debonding test. Representative stress-strain curves are shown in figure 3. All curves are characterized by a stress peak at small strain followed by a continuous decrease until a sharp decrease of stress occurs from $\sigma_{0} \approx 10^{5} \mathrm{~Pa}$ to $\sigma_{0} \approx 5.10^{4}-10^{4}$ $\mathrm{Pa}$. At larger deformation, $\sigma_{0}$ continues to decrease more or less steeply in the case of copolymers while $\sigma_{0}$ becomes rapidly too low to be detected for PnBAX polymers.

This shape of the stress-strain curve is usually observed in probe-tack tests carried out on confined viscous or viscoelastic films ${ }^{31,32}$, i.e. when the contact radius $r_{0}$ is much higher than the initial thickness $h_{0}\left(r_{0} / h_{0}=50\right.$ in our case $)$. At the beginning of the debonding test, high stresses are measured due to the low compliance of the thin layer ${ }^{32}$. As cavities grow from the probe surface into the film (see figure S6), stress relaxes through the film and the spring-like response of the apparatus induces a large deformation of the adhesive layer ${ }^{33,34,35}$. At the end of the adhesion test, the fracture of the walls between cavities allows the pressure between the ambient atmosphere and the internal cavities to equilibrate ${ }^{32,36}$ and when bubbles coalesce, the stress drops. The residual stress measured after the equilibration of pressure results from the uniaxial elongation of remaining fibrils between the steel probe and the glass slide.

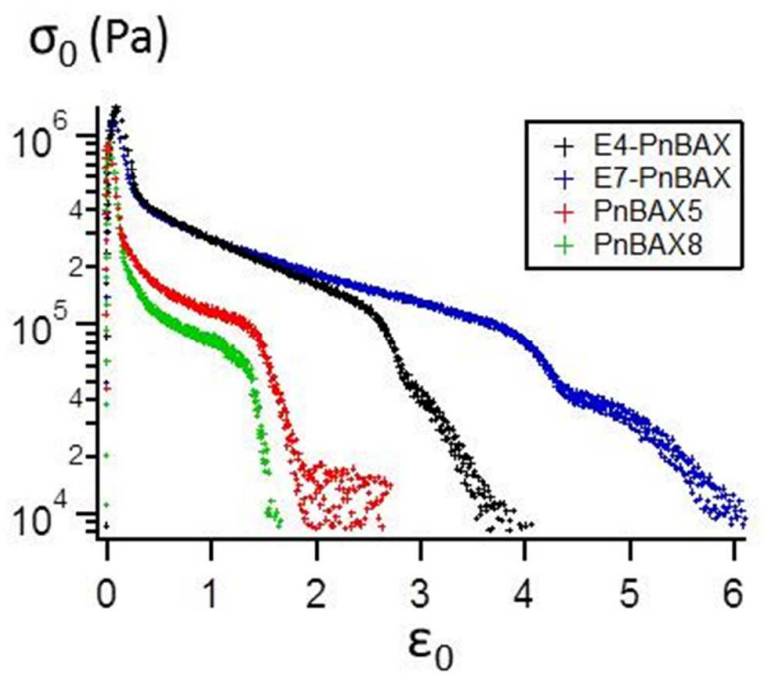

Figure 3: Probe tack test at $V_{d e b}=100 \mu \mathrm{m} / \mathrm{s}$ and room temperature for PnBAX5,PnBAX8, E7-PnBAX and E4-PnBAX.

Significant differences are observed between the PnBAX materials and the supramolecular copolymers. The maximal nominal strain $\varepsilon_{\max }$, measured at the minimum of stress detected by the load cell (i.e. $\sigma_{0} \approx 10^{4} \mathrm{~Pa}$ ), is $\varepsilon_{\max }=5.5 \pm 0.5$ and $\varepsilon_{\max }=3.8 \pm 0.3$ for E7-PnBAX and E4PnBAX respectively while $\varepsilon_{\max }$ is lower than 2 for PnBAX5 and PnBAX8. The adhesion energy $W_{\text {adh }}$ is close to $90 \mathrm{~J} / \mathrm{m}^{2}$ for copolymers while $W_{\text {adh }}$ is around $30 \mathrm{~J} / \mathrm{m}^{2}$ for PnBAX5 and PnBAX8 (see table 3). Fibrils stretch more before breaking and the detachment of the steel probe requires more energy than for PnBAX5 and PnBAX8. However the debonding is still cohesive for both types of materials. This result shows that the combined presence of the bisurea moiety and of the glycidyl methacrylate comonomer increase the fibril stability in extension suggesting some level of interaction. 
In order to strengthen fibrils further at large deformations, a chain extending step can then be added to the experimental protocol. Before the casting process, a diamine is added to the solution of supramolecular copolymers and this diamine can react with the epoxy groups of the copolymer chains during the post-casting drying steps (see figures S3-S5). The influence of the concentration of cross-linkers on rheology and adhesion of copolymers is studied in the following part.

\section{Copolymers cured by diamine}
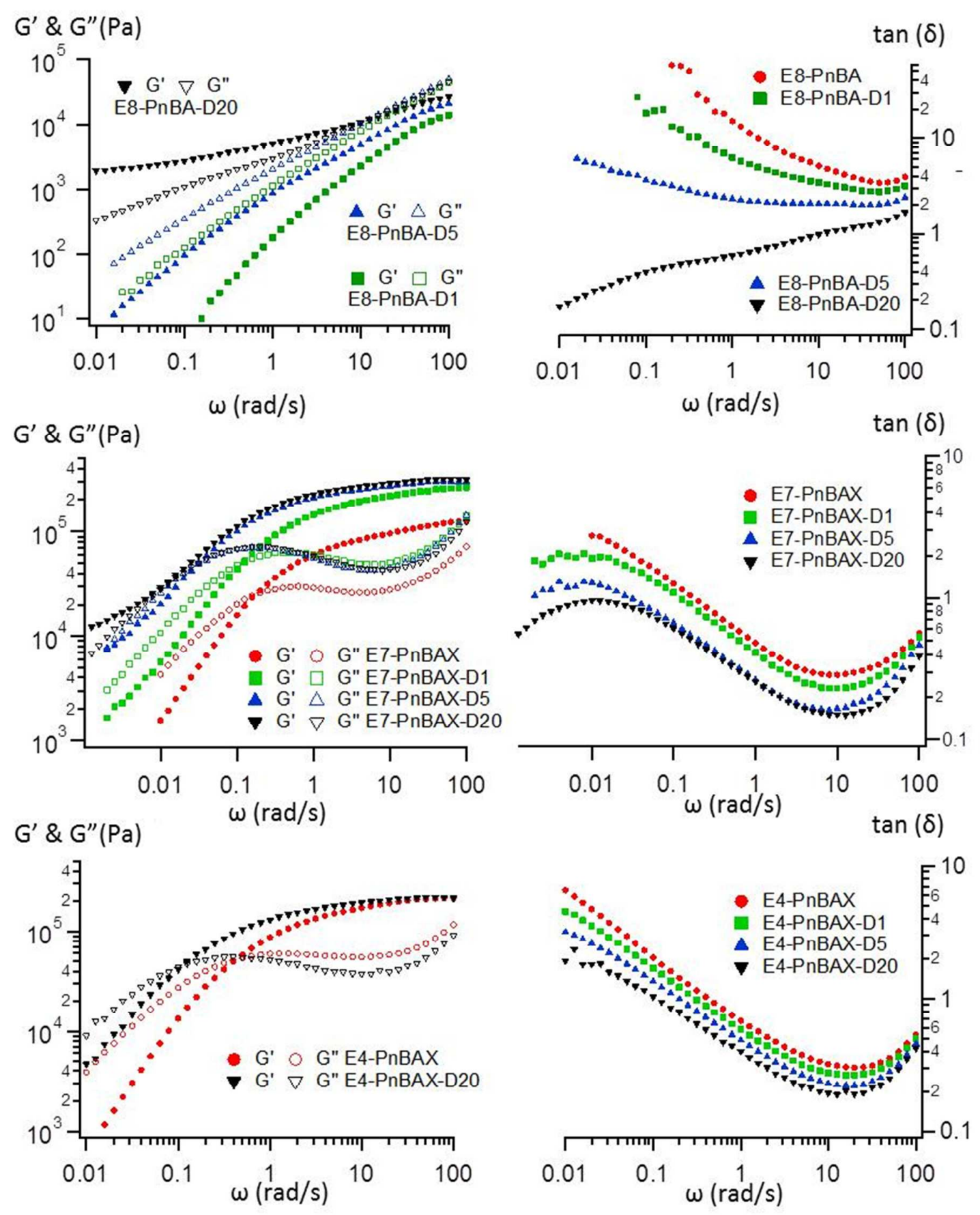
Figure 4: Variation of $G^{\prime}, G^{\prime \prime}$ (left) and $\tan \delta$ (right) with frequency at $25^{\circ} \mathrm{C}$ in the linear regime for copolymers and for their respective blends with diamine (after curing).

In figure 4, the linear viscoelastic properties of the copolymers cured with different concentrations of cross-linkers [D] are compared with that of the respective initial copolymer (without diamine) at $25^{\circ} \mathrm{C}$. The frequency dependence of the damping factor $\tan \delta$ is especially useful to examine the cross-linking process, from the extension of the polymer chains to the formation of a polymer network.

For the reference copolymer E8-PnBA which contains epoxy groups but no bis-urea, a typical viscoelastic fluid response with a broad spectrum of relaxation times is observed for both E8PnBA-D1 and E8-PnBA-D5 suggesting that only chain extension occurred. At a fixed frequency $\omega$, the decrease of $\tan \delta$ with increasing [D] characterizes the increase of the relaxation times and thus, the degree of extension of the polymer chains ${ }^{37,38}$ via the coupling reaction. On the other hand, for E8-PnBA-D20, a gel-like response is observed: the storage modulus $G$ ' is higher than the viscous modulus $G$ ', for all $\omega$ and $\tan \delta$ increases with $\omega$. This behavior reveals the presence of a percolating polymer network through the material.

As observed for the E8-PnBA, the chain extending process makes the E7-PnBAX copolymers more elastic in the linear regime: the storage modulus $G$ ' increases with cross-linker concentration [D] for all frequencies while the damping factor $\tan \delta$ decreases. Like E7PnBAX, E7-PnBAX-D1 shows a viscoelastic response characterized by an elastic plateau for $\omega>\omega_{c}=0.1 \mathrm{rad} / \mathrm{s} \pm 0.03$ and a viscous regime at lower $\omega$. For E7-PnBAX-D5 and E7PnBAX-D20, the decrease of the damping factor $\tan \delta$ with $\omega$ seems to reveal a second elastic plateau at low frequency and thus, an elastic behavior at long time scales. The existence of this second plateau is confirmed by oscillatory experiments carried out at $T=80^{\circ} \mathrm{C}$ (see figure S7).

The frequency dependence of $G^{\prime}$ and $G^{\prime \prime}$ at $25^{\circ} \mathrm{C}$ is also shown for several supramolecular resins synthesized from E4-PnBAX in figure 4. As expected, $G$ ' increases with cross-linker concentration [D] for all frequencies while tan $\delta$ decreases. Unlike E7-PnBAX-based resins however, all resins synthesized from E4-PnBAX are viscoelastic fluids at $25^{\circ} \mathrm{C}$. Their spectrum of relaxation times is divided into an elastic plateau at high $\omega$ and a terminal zone at low $\omega$. The longest relaxation time determined by $\tan \delta=1$ increases with cross-linker concentration, from $\tau=1 / \omega_{c}=3 s \pm 1$ to $\tau=10 s \pm 1$. The absence of a second plateau at low $\omega$ is supported by the frequency dependence of $G$ ' and $G$ '” at $80^{\circ} \mathrm{C}$ (see figure S8).

The resins cured from the three types of copolymers at different concentrations of cross-linker [D] were also characterized by probe-tack tests at $V_{d e b}=100 \mu \mathrm{m} / \mathrm{s}$ and room temperature. Representative stress-strain curves are shown in figure 5 while the debonding energy and the mode of failure (adhesive/cohesive) are reported in table 3.

For the reference copolymer E8-PnBA which does not contain bis-urea groups, the stressstrain curves show a similar shape for all investigated concentrations of diamine. As observed for copolymers, all curves are characterized by a stress peak at small strain and a continuous decrease of stress at higher strain. However, a significant increase of the adhesion energy is 
observed with increasing concentration of diamine: $W_{\text {adh }}$ is measured at $40 \mathrm{~J} / \mathrm{m}^{2} \pm 7$ for E8PnBA-D20 while $W_{\text {adh }}$ is below $20 \mathrm{~J} / \mathrm{m}^{2}$ at lower [D]. Unlike E8-PnBA-D1 and E8-PnBA-D5, an adhesive failure occurs for E8-PnBA-D20 analogously to commercial soft adhesives, no polymer is left on the probe after the adhesion test (see figure S9). The maximal strain $\varepsilon_{\max }$ also increases with cross-linker concentration, i.e $\varepsilon_{\max }=1.4 \pm 0.1$ for E8-PnBA-D1 $2.0 \pm 0.2$ for E8-PnBA-D5 and $3.3 \pm 0.3$ for E8-PnBA-D20. This is the classical effect that one would expect when low molecular weight linear non-interacting polymers are chain extended and entanglements and a light level of crosslinking are formed ${ }^{31}$.
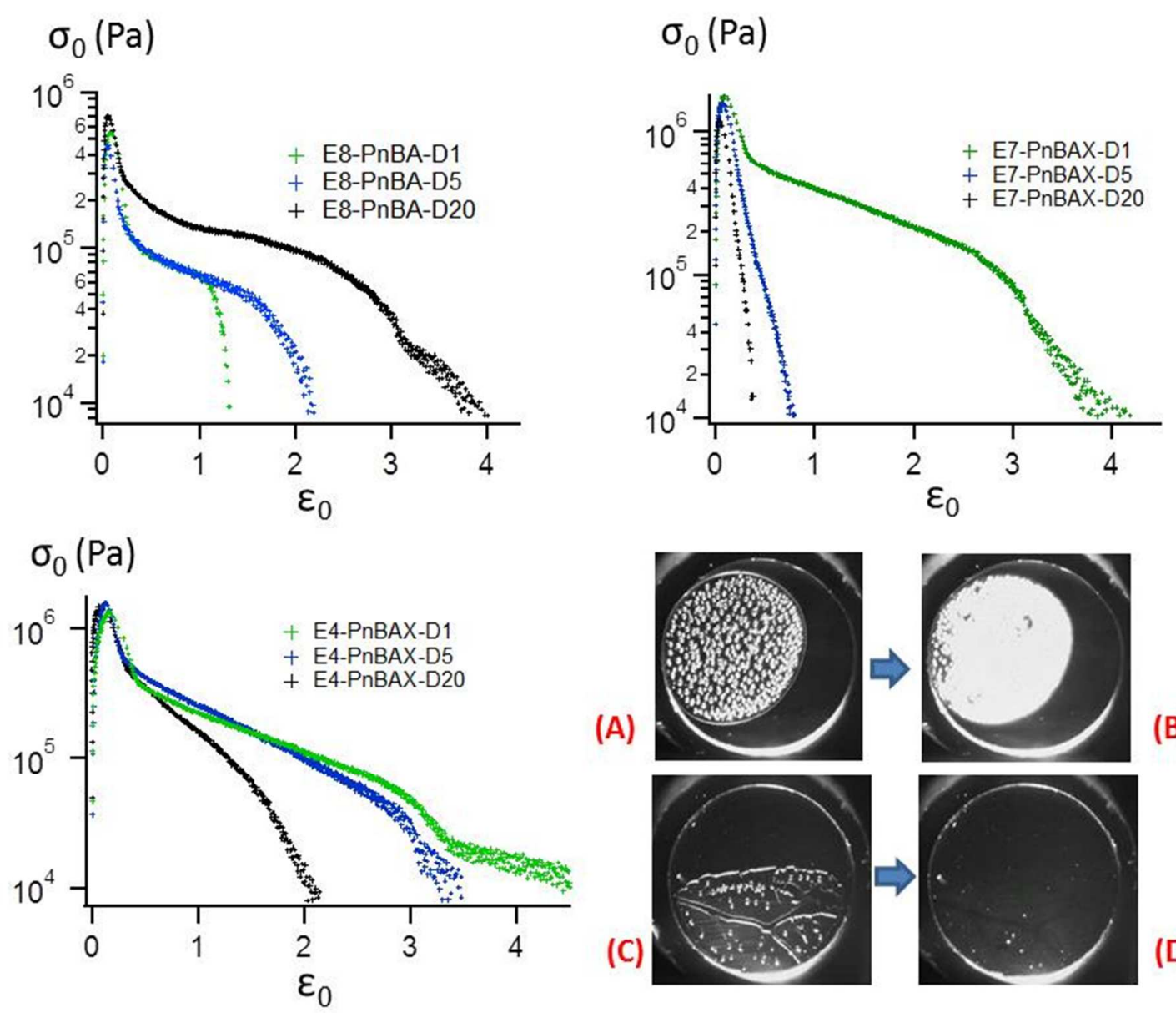

(A)
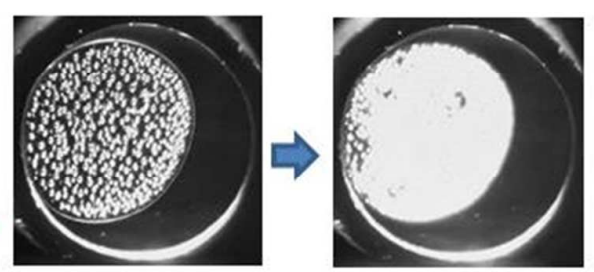

(B)

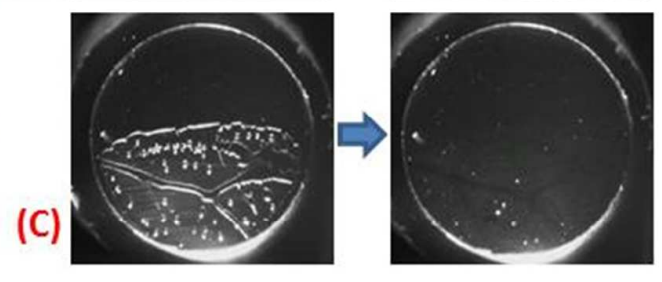

(D)

Figure 5: Stress-strain curves of probe tack test at $V_{d e b}=100 \mu \mathrm{m} / \mathrm{s}$ for E8-PnBA, E7-PnBAX, E4PnBAX and the respective resins synthesized at different diamine concentrations. Debonding mechanisms observed through the glass substrate for E7-PnBAX-D1 (A and B) and E7-PnBAX-D20 (C and D). Cavitation and uniaxial deformation of the fibrils (A) revealed by light scattering. Image (B) of the surface of the E7-PnBAX-D1 layer after the adhesion test. Cavitation and growth of cracks during the adhesion test (C), photo of the surface of the E7-PnBAX-D20 after the test (D).

For the E7-PnBAX-based resins, the steel probe detaches by adhesive failure for the three investigated diamine concentrations. Stress-strain curves are reduced to a high stress peak for the two most cross-linked materials while stress decreases continuously up to $\varepsilon_{\max }=3.9 \pm 0.4$ for E7-PnBAX-D1 (see figure 5). The reduced deformation observed for E7-PnBAX-D5 and 
E7-PnBAX-D20 entails low debonding energies $\left(W_{a d h}<50 \mathrm{~J} / \mathrm{m}^{2}\right)$ while $W_{\text {adh }}$ is close to $100 \mathrm{~J} / \mathrm{m}^{2}$ for E7-PnBAX-D1. The change of shape of the stress-strain curves reveals a transition in debonding mechanism from bulk cavitation to interfacial cracks ${ }^{39,40}$, as illustrated by the images of the debonding tests observed through the thin adhesive film ${ }^{29}$ in figure 5 (see also S10 and S11). For E7-PnBAX-D1, the cavitation near the surface of the steel probe is followed by the stretch of the walls between cavities. Light scattering enhances the large deformation of the adhesive layer up to the complete detachment of the probe (image 5.A). In this case, the surface of the thin adhesive layer remains highly deformed after the adhesion test (image 5.B). For E7-PnBAX-D20, cracks and cavities appear at the beginning of the debonding test and grow along the interface between the steel probe and the adhesive (image 5.C). The surface of the thin layer is still smooth and undamaged after the adhesion test (image 5.D).

For the intermediate case of the E4-PnBAX-based resins, stress-strain curves are all characterized by a high stress peak at small strain and a continuous decrease of stress at large strains. Cavitation and fibrillation mechanisms are observed for all diamine concentration, consistent with the rheology of figure 4 but an adhesive failure is only observed for the highest investigated concentration of diamine. The probe detaches from the adhesive film at lower strains $\left(\varepsilon_{\max } \leq 2\right)$ for E4-PnBAX-D20 than for the other resins $\left(\varepsilon_{\max } \geq 3\right)$. This reduced deformation entails a lower debonding energies $\left(W_{a d h}=54 \pm 6 \mathrm{~J} / \mathrm{m}^{2}\right)$ for E4-PnBAX-D20 than for the other resins $\left(W_{a d h} \sim 90 \mathrm{~J} / \mathrm{m}^{2}\right)$.

\begin{tabular}{|c|c|c|c|c|c|}
\hline & Material & $W_{a d h}\left(\mathrm{~J} / \mathbf{m}^{2}\right)$ & Mode of failure & $\varepsilon_{\max }$ & $\begin{array}{c}\operatorname{Tan} \delta / G \\
\left(M^{\prime} a^{-1}\right)\end{array}$ \\
\hline \multirow{4}{*}{$\begin{array}{l}\text { Without } \\
\text { Diamine }\end{array}$} & PnBAX5 & $33 \pm 6$ & Cohesive & $1.9 \pm 0.2$ & 7 \\
\hline & PnBAX8 & $30 \pm 4$ & Cohesive & $1.6 \pm 0.1$ & 180 \\
\hline & E7-PnBAX & $90 \pm 14$ & Cohesive & $5.5 \pm 0.5$ & 3 \\
\hline & E4-PnBAX & $87 \pm 9$ & Cohesive & $3.8 \pm 0.3$ & 2 \\
\hline \multirow{9}{*}{ With Diamine } & E8-PnBA-D1 & $13 \pm 1$ & Cohesive & $1.4 \pm 0.1$ & 2500 \\
\hline & E8-PnBA-D5 & $18 \pm 5$ & Cohesive & $2.0 \pm 0.2$ & 600 \\
\hline & E8-PnBA-D20 & $40 \pm 7$ & Adhesive & $3.3 \pm 0.3$ & 100 \\
\hline & E7-PnBAX-D1 & $104 \pm 10$ & Adhesive & $3.9 \pm 0.4$ & 1 \\
\hline & E7-PnBAX-D5 & $48 \pm 5$ & Adhesive & $0.8 \pm 0.1$ & 0.6 \\
\hline & E7-PnBAX-D20 & $17 \pm 2$ & Adhesive & $0.4 \pm 0.1$ & 0.6 \\
\hline & E4-PnBAX-D1 & $81 \pm 10$ & Cohesive & $4.5 \pm 0.4$ & 2 \\
\hline & E4-PnBAX-D5 & $90 \pm 6$ & Cohesive & $3.2 \pm 0.3$ & 1 \\
\hline & E4-PnBAX-D20 & $54 \pm 6$ & Adhesive & $1.8 \pm 0.2$ & 1 \\
\hline
\end{tabular}


Table 3: Debonding energy $\left(W_{a d h}\left(\mathrm{~J} / \mathrm{m}^{2}\right)\right)$ measured by a probe tack test at $V_{d e b}=100 \mu \mathrm{m} / \mathrm{s}$ and at room temperature. $G^{\prime}$ and $\tan \delta$ are measured at $25^{\circ} \mathrm{C}$ and $6.28 \mathrm{rad} / \mathrm{s}$.

\section{Discussion}

\section{Supramolecular Polymer network}

Linear rheology is especially illuminating to identify the different molecular mechanisms controlling the deformation of materials. In figure 2, the comparison of the viscoelastic properties of the three copolymers clearly highlights the role of the supramolecular interactions in the rheology of the bis-urea center-functionalized copolymers. The contrast between their viscoelastic character and the liquid-like behavior of E8-PnBA suggests a strong increase of the terminal time of the copolymer chains due to the presence of urea groups. The terminal time of the copolymers is clearly higher than that of centerfunctionalized poly(butylacrylate) (PnBAX5 and PnBAX8) which have a higher density of urea groups.

These results reveal that the linear rheological properties of the supramolecular copolymers are not fully controlled by interactions between bis-urea groups but probably also by interactions of bis-urea with the side chains, particularly with glycidyl methacrylate (GMA) comonomers. Complementary investigations were carried out at low concentrations in non polar solvent (see figure S12 in SI) and demonstrated that GMA is a stronger hydrogen bond competitor than butyl methacrylate. This effect is probably due to the fact that the glycidyl methacrylate monomer contains two hydrogen bond acceptor groups (carbonyl and epoxy) that may interact cooperatively with the bis-urea moiety. Although no SAXS data could confirm this assumption, this change in rheological properties suggests a change of nanostructure induced by the incorporation of glycidyl methacrylate monomers at the end of the bis-urea center-functionalized polymer chains. As illustrated in figure 6 , the change from monofunctional to multifunctional chains probably entails the transition from comb-shaped aggregates $^{8,13}$ towards a transient polymer network.

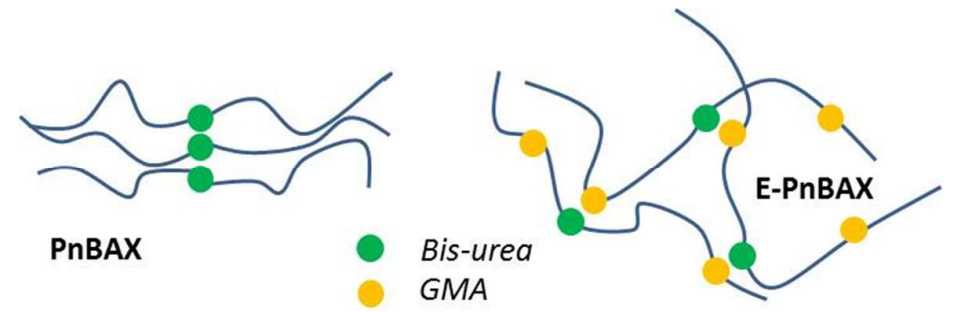

Figure 6: schemes of the supramolecular aggregates and the transient network in PnBAX and EPnBAX copolymers, respectively.

This polymer network is dynamic since these supramolecular interactions cannot prevent the copolymer chains from flowing at long time scales. A solid-like response, characterized by an elastic plateau at low frequency, requires a chain-extension as observed for E7-PnBAX-D5 and E7-PnBAX-D20 in figure 4. The solid or liquid-like behavior of these slightly cross- 
linked copolymers depends on the cross-linker density as well as the level of functionalization of the copolymers, as revealed by the differences in rheology between E8-PnBA-D5, E7PnBAX-D5 and E4-PnBAX-D20. Among these three materials, an elastic plateau at low frequency is only observed for E7-PnBAX-D5 while this material possesses the lowest average number of cross-linker per copolymer. Unlike E7-PnBAX, E8-PnBA is not functionalized by a bis-urea moiety and thus, the urea-epoxy interactions are absent in E8PnBA-D5. As for E4-PnBAX, the density of glycidyl methacrylate monomers per chain is lower than for E7-PnBAX and thus, weak interactions between bis-urea and carbonyl groups of butylacrylate ${ }^{26}$ may be more favored than interactions between bis-urea and GMA in E4PnBAX-D20 than in E7-PnBAX-D5. In other words, the clusters or dimers formed via strong non covalent bonds are expected to be less dense in E4-PnBAX-D20 than in E7-PnBAX-D5. These results underline the role of the supramolecular interactions in the polymer network, which has a dual nature (chemical and physical).

\section{From Cohesive to Adhesive Failure}

The strengthening of the polymer matrix by supramolecular interactions observed in linear rheology is confirmed by the high stress peaks $\left(\sigma_{\max }>1 \mathrm{MPa}\right)$ and the high adhesion energy $\left(W_{a d h} \sim 90 \mathrm{~J} / \mathrm{m}^{2}\right)$ measured for E7-PnBAX and E4-PnBAX in probe-tack tests. However, the supramolecular interactions are not sufficient to prevent the failure of the fibrils at large deformations and thus, to induce an adhesive failure for the investigated debonding velocity and temperature. This suggests that these materials soften significantly with increasing strain as demonstrated for similar tris-urea functionalized supramolecular materials $^{15}$.

A classical method to obtain a clean removal of the PSA is to create a strain hardening in extensional rheology ${ }^{41}$. In turn such a strain hardening can be obtained by introducing long chain branching, a slight crosslinking to the polymer linear chain ${ }^{3,19,42}$ or several dynamic stickers per chain ${ }^{22}$. In our particular system it is possible to slightly covalently cross-link the polymer chains $^{43}$, as shown by E8-PnBA-D20. Since several epoxy groups per chain are present per copolymer chain, the cross-linking of the E8-PnBA copolymer is expected to form a branched covalent polymer network ${ }^{42,44}$. As previously reported in the literature ${ }^{16}$, the formation of a covalent polymer network is not sufficient for the elaboration of a PSA and non covalent interactions are often required to couple high debonding energy and a full detachment of the adhesive. This is clearly illustrated here by the difference between E8PnBA-D20 and E7-PnBAX-D1. Although an adhesive failure was observed for both materials, the stress peak and the debonding energy were much higher for the latter $\left(\sigma_{\max } \sim\right.$ $1.2 \mathrm{MPa}$ and $\left.W_{a d h} \sim 100 \mathrm{~J} / \mathrm{m}^{2}\right)$ than for the former $\left(\sigma_{\max } \sim 0.6 \mathrm{MPa}\right.$ and $\left.W_{a d h} \sim 40 \mathrm{~J} / \mathrm{m}^{2}\right)$. While E8-PnBA-D20 looks like a soft elastic gel $\left(G^{\prime} \sim 10 \mathrm{kPa}\right)$, the supramolecular interactions in E7-PnBAX-D1 gives a strong viscoelastic character to the polymer network and thus induces a high energy dissipation at large deformations.

The solid-like behavior observed for E7-PnBAX-D5 and E7-PnBAX-D20 in the adhesion tests reveals that the [D] range where interesting adhesive properties can be obtained on steel is quite limited for the E7-PnBAX-based resins. The strong elastic character of these networks 
is confirmed by the ratio $\tan \left(\delta\left(\omega_{\text {adh }}\right)\right) / G^{\prime}\left(\omega_{\text {adh }}\right) \sim 0.6 \mathrm{MPa}^{-1}$ measured at the equivalent angular frequency of the adhesion test $\omega_{a d h}=2 \pi V_{d e b} / h_{0}=6.28 \mathrm{rad} / \mathrm{s}$ These ratios are almost ten times lower than the critical ratio $\left[(\tan \delta) / G^{\prime}\right]_{c} \sim 5 \mathrm{MPa}^{-1}$ proposed by Deplace et al ${ }^{45}$, below which debonding by interfacial cracks may occur on steel substrate.

The characterization of the E4-PnbAX-based resins reveals that a better balance between low viscoelastic moduli and strain hardening can be obtained by modifying the chemical architecture of the supramolecular polymer network. The decrease of the number of glycidyl methacrylate monomers per chain allowed to significantly modify the rheological and adhesive properties. For the three investigated diamine concentrations E4-PnBAX-based resins are more dissipative at small strains than the resins synthesized from E7-PnBAX ((tan $\delta) / G^{\prime}\left(\omega=\omega_{\text {adh }}\right) \geq 1 \mathrm{MPa}^{-1}$ for all [D] $)$ and in adhesion tests, all E4-PnBAX-based resins show non negligible bulk deformation and debonding energy $\left(\varepsilon_{\max } \geq 2\right.$ and $W_{\text {adh }} \geq 60 \mathrm{~J} / \mathrm{m}^{2}$ for all [D]). The transition from cohesive to adhesive failure is also shifted to higher cross-linker density (between 0.1 and $0.4 \%$ ) making the synthesis process more flexible. The influence of the number of glycidyl methacrylate per chain on the adhesive properties highlights their dual role both as stickers and as chain extenders (by the reaction with diamine).

Our study, which focused on a reduced range of chemical parameters and only used linear rheology and probe tack as characterization tools, is not intended to be exhaustive but is a first proof of concept study for the design of supramolecular PSA based on a simple two-step process. The first step consists in synthesizing low molecular weights supramolecular polymers, which can be easily processed into thin films by classical methods (solvent casting, extrusion...). The second step consists in modifying the architecture of the polymer chains by cross-linking, chain extending or branching by an external stimulus (heat in our case). By an appropriate functionalization of the low molecular weight polymer, these two steps can be complementary to finely tune the linear and non linear rheology in order to optimize the adhesive properties. It also suggests that synthesizing slightly higher molecular weight polymers with multiple stickers per chain might be a viable strategy for a one-step synthesis. Finally it should be noted that this type of chemistry could in principle be obtained with polyurethanes. However there are two major differences between our systems and classic polyurethanes: the urethane functionality is a weaker hydrogen bonding moiety than the urea providing less dissipation, and the polyethers typically used as soft blocks in PU are much too entangled to be useful as PSA. This makes in principle polyurethanes much more suitable for elastomers than for soft and sticky PSA.

\section{Conclusion}

A new strategy to synthesize supramolecular Pressure Sensitive Adhesives (PSA) was explored. Poly(butylacrylate -co- glycidyl methacrylate) copolymers functionalized by a bisurea sticker in the middle were synthesized and then, slightly chain-extended by reacting with a diamine. The optimization of the rheological and adhesive properties of these materials for PSA-like applications was illustrated by studying the influence of the ratio of glycidyl methylacrylate and diamine per chain at a fixed molecular weight $\left(\mathrm{M}_{\mathrm{n}}=10 \mathrm{~kg} / \mathrm{mol}\right)$. Our results showed that the non covalent interactions between copolymers via bis-urea moieties and 
GMA comonomers give rise to a supramolecular polymer network and lead to dissipative properties at small and large deformations. Combining high debonding energies and clean removal requires to slightly cross-link the chains of the low molecular weight copolymers with an appropriate amount of diamine. At high diamine contents, films are too elastic to show high debonding energies. From theses preliminary results, further works on a larger range of chemical architectures would help to design PSA with a very low or no insoluble fraction (gel) ${ }^{45}$. The reduction of the gel fraction, while keeping good adhesive properties, is a key issue for the elaboration of smart and recyclable adhesives.

\section{Associated Content (Supporting Information)}

Synthesis details, characterization by NMR and SEC for copolymers; DSC characterization for the curing step of resins; small amplitude oscillatory shear tests carried out at $80^{\circ} \mathrm{C}$ for copolymers and resins; debonding pictures of probe-tack tests; complementary investigations on supramolecular interactions in dilute solutions with a capillary viscometer.

\section{Acknowledgments}

This work was financially supported by the ANR SUPRADHESION program (Project ANR10-BLAN-0801).

\section{References}

(1) Creton, C. Pressure-Sensitive-Adhesives : An Introductory Course. MRS Bull. 2003, 28 (6), 434-439.

(2) PSTC. Test Methods for Pressure Sensitive Adhesive Tapes, 13th ed.; Pressure Sensitive Tape Council: Oakbrook Terrace.; USA, 2000.

(3) Gower, M. D.; Shanks, R. A. The Effect of Chain Transfer Agent Level on Adhesive Performance and Peel Master-Curves for Acrylic Pressure Sensitive Adhesives. Macromol. Chem. Phys. 2004, 205 (16), 2139-2150.

(4) Lopez, A.; Degrandi-Contraires, E.; Canetta, E.; Creton, C.; Keddie, J. L.; Asua, J. M. Waterborne Polyurethane-Acrylic Hybrid Nanoparticles by Miniemulsion Polymerization: Applications in Pressure-Sensitive Adhesives. Langmuir 2011, 27 (7), 3878-3888.

(5) Feldstein, M. M.; Dormidontova, E. E.; Khokhlov, A. R. Pressure Sensitive Adhesives Based on Interpolymer Complexes. Prog. Polym. Sci. 2015, 42, 79-153.

(6) Heinzmann, C.; Weder, C.; de Espinosa, L. M. Supramolecular Polymer Adhesives: Advanced Materials Inspired by Nature. Chem Soc Rev 2016, 45 (2), 342-358.

(7) Aida, T.; Meijer, E. W.; Stupp, S. I. Functional Supramolecular Polymers. Science 2012, 335 (6070), 813-817.

(8) Courtois, J.; Baroudi, I.; Nouvel, N.; Degrandi, E.; Pensec, S.; Ducouret, G.; Chanéac, C.; Bouteiller, L.; Creton, C. Supramolecular Soft Adhesive Materials. Adv. Funct. Mater. 2010, 20 (11), 1803-1811.

(9) Cheng, S.; Zhang, M.; Dixit, N.; Moore, R. B.; Long, T. E. Nucleobase Self-Assembly in Supramolecular Adhesives. Macromolecules 2012, 45 (2), 805-812.

(10) Faghihnejad, A.; Feldman, K. E.; Yu, J.; Tirrell, M. V.; Israelachvili, J. N.; Hawker, C. J.; Kramer, E. J.; Zeng, H. Adhesion and Surface Interactions of a Self-Healing 
Polymer with Multiple Hydrogen-Bonding Groups. Adv. Funct. Mater. 2014, 24 (16), 2322-2333.

(11) McEachran, M. J.; Trant, J. F.; Sran, I.; de Bruyn, J. R.; Gillies, E. R. Carboxylic AcidFunctionalized Butyl Rubber: Synthesis, Characterization, and Physical Properties. Ind. Eng. Chem. Res. 2015, 54 (17), 4763-4772.

(12) Ahn, Y.; Jang, Y.; Selvapalam, N.; Yun, G.; Kim, K. Supramolecular Velcro for Reversible Underwater Adhesion. Angew. Chem. Int. Ed. 2013, 52 (11), 3140-3144.

(13) Véchambre, C.; Callies, X.; Fonteneau, C.; Ducouret, G.; Pensec, S.; Bouteiller, L.; Creton, C.; Chenal, J.-M.; Chazeau, L. Microstructure and Self-Assembly of Supramolecular Polymers Center-Functionalized with Strong Stickers. Macromolecules 2015, 48 (22), 8232-8239.

(14) Callies, X.; Véchambre, C.; Fonteneau, C.; Pensec, S.; Chenal, J.-M.; Chazeau, L.; Bouteiller, L.; Ducouret, G.; Creton, C. Linear Rheology of Supramolecular Polymers Center-Functionalized with Strong Stickers. Macromolecules 2015, 48 (19), 7320 7326.

(15) Callies, X.; Fonteneau, C.; Pensec, S.; Bouteiller, L.; Ducouret, G.; Creton, C. Adhesion and Non-Linear Rheology of Supramolecular Tri-Urea Center-Functionalized Poly(butylacrylate) Investigated by Probe-Tack Tests. Soft Matter 2016, 12, 71747185.

(16) Lindner, A.; Lestriez, B.; Mariot, S.; Creton, C.; Maevis, T.; Lühmann, B.; Brummer, R. Adhesive and Rheological Properties of Lightly Crosslinked Model Acrylic Networks. J. Adhes. 2006, 82 (3), 267-310.

(17) Degrandi-Contraires, E.; Lopez, A.; Reyes, Y.; Asua, J. M.; Creton, C. High-ShearStrength Waterborne Polyurethane/Acrylic Soft Adhesives. Macromol. Mater. Eng. 2013, 298 (6), 612-623.

(18) Creton, C.; Ciccotti, M. Fracture and Adhesion of Soft Materials: A Review. Rep. Prog. Phys. Phys. Soc. G. B. 2016, 79 (4), 046601.

(19) Lentzakis, H.; Vlassopoulos, D.; Read, D. J.; Lee, H.; Chang, T.; Driva, P.; Hadjichristidis, N. Uniaxial Extensional Rheology of Well-Characterized Comb Polymers. J. Rheol. 2013, 57 (2), 605-625.

(20) Ruymbeke, E. van; Nielsen, J.; Hassager, O. Linear and Nonlinear Viscoelastic Properties of Bidisperse Linear Polymers: Mixing Law and Tube Pressure Effect. $J$. Rheol. 2010, 54 (5), 1155-1172.

(21) Goldansaz, H.; Fustin, C.-A.; Wübbenhorst, M.; van Ruymbeke, E. How Supramolecular Assemblies Control Dynamics of Associative Polymers: Toward a General Picture. Macromolecules 2016, 49 (5), 1890-1902.

(22) Shabbir, A.; Goldansaz, H.; Hassager, O.; van Ruymbeke, E.; Alvarez, N. J. Effect of Hydrogen Bonding on Linear and Nonlinear Rheology of Entangled Polymer Melts. Macromolecules 2015, 48 (16), 5988-5996.

(23) Vendamme, R.; Olaerts, K.; Gomes, M.; Degens, M.; Shigematsu, T.; Eevers, W. Interplay Between Viscoelastic and Chemical Tunings in Fatty-Acid-Based Polyester Adhesives: Engineering Biomass toward Functionalized Step-Growth Polymers and Soft Networks. Biomacromolecules 2012, 13 (6), 1933-1944.

(24) Li, A.; Li, K. Pressure-Sensitive Adhesives Based on Epoxidized Soybean Oil and Dicarboxylic Acids. ACS Sustain. Chem. Eng. 2014, 2 (8), 2090-2096.

(25) Isare, B.; Pensec, S.; Raynal, M.; Bouteiller, L. Bisurea-Based Supramolecular Polymers : From Structure to Properties. C R Chim. 2016, 19, 148-156.

(26) Fonteneau, C.; Pensec, S.; Bouteiller, L. Versatile Synthesis of Reversible CombShaped Supramolecular Polymers. Polym. Chem. 2014, 5 (7), 2496-2505. 
(27) Callies, X.; Fonteneau, C.; Véchambre, C.; Pensec, S.; Chenal, J.-M.; Chazeau, L.; Bouteiller, L.; Ducouret, G.; Creton, C. Linear Rheology of Bis-Urea Functionalized Supramolecular Poly(butylacrylate)s: Part I - Weak Stickers. Polymer 2015, 69, 233240.

(28) Brandrup, J.; Immergut, E. .; Grulke, E. .; Abe, A.; Bloch, D. R. Polymer Handbook (4th Edition); John Wiley\&Sons, 1999.

(29) Lakrout, H.; Sergot, P.; Creton, C. Direct Observation of Cavitation and Fibrillation in a Probe Tack Experiment on Model Acrylic Pressure-Sensitive-Adhesives. J. Adhes. 1999, 69 (3-4), 307-359.

(30) Villey, R.; Creton, C.; Cortet, P.-P.; Dalbe, M.-J.; Jet, T.; Saintyves, B.; Santucci, S.; Vanel, L.; Yarusso, D. J.; Ciccotti, M. Rate-Dependent Elastic Hysteresis during the Peeling of Pressure Sensitive Adhesives. Soft Matter 2015, 11 (17), 3480-3491.

(31) Lakrout, H.; Creton, C.; Ahn, D.; Shull, K. R. Influence of Molecular Features on the Tackiness of Acrylic Polymer Melts. Macromolecules 2001, 34 (21), 7448-7458.

(32) Poivet, S.; Nallet, F.; Gay, C.; Teisseire, J.; Fabre, P. Force Response of a Viscous Liquid in a Probe-Tack Geometry: Fingering versus Cavitation. Eur. Phys. J. E 2004, 15 (2), 97-116.

(33) Francis, B. A.; Horn, R. G. Apparatus-Specific Analysis of Fluid Adhesion Measurements. J. Appl. Phys. 2001, 89 (7), 4167-4174.

(34) Poivet, S.; Nallet, F.; Gay, C.; Fabre, P. Cavitation-Induced Force Transition in Confined Viscous Liquids under Traction. EPL Europhys. Lett. 2003, 62 (2), 244-250.

(35) Tirumkudulu, M.; Russel, W. B.; Huang, T. J. On the Measurement of "tack" for Adhesives. Phys. Fluids 2003, 15 (6), 1588-1605.

(36) Tanguy, F.; Nicoli, M.; Lindner, A.; Creton, C. Quantitative Analysis of the Debonding Structure of Soft Adhesives. Eur. Phys. J. E 2014, 37 (1), 1-12.

(37) Rubinstein, M.; Colby, R. H.; Gillmor, J. R. rubinstein1989. Springer Ser. Chem. Phys. 1989, 51, 66-74.

(38) Winter, H. H.; Chambon, F. Analysis of Linear Viscoelasticity of a Crosslinking Polymer at the Gel Point. J. Rheol. 1986, 30, 367-382.

(39) Crosby, A. J.; Shull, K. R.; Lakrout, H.; Creton, C. Deformation and Failure Modes of Adhesively Bonded Elastic Layers. J. Appl. Phys. 2000, 88 (5), 2956-2966.

(40) Nase, J.; Lindner, A.; Creton, C. Pattern Formation during Deformation of a Confined Viscoelastic Layer: From a Viscous Liquid to a Soft Elastic Solid. Phys. Rev. Lett. 2008, 101 (7).

(41) Glassmaker, N. J.; Hui, C. Y.; Yamaguchi, T.; Creton, C. Detachment of Stretched Viscoelastic Fibrils. Eur. Phys. J. E 2008, 25 (3), 253-266.

(42) Zosel, A. Effect of Cross-Linking on Tack and Peel Strength of Polymers. J. Adhes. 1991, 34, 201-209.

(43) Zosel, A.; Ley, G. Influence of Crosslinking on Structure, Mechanical Properties, and Strength of Latex Films. Macromolecules 1993, 26 (9), 2222-2227.

(44) Cañamero-Martínez, P.; Fernández-García, M.; Fuente, J. L. de la. Rheological Cure Characterization of a Polyfunctional Epoxy Acrylic Resin. React. Funct. Polym. 2010, 70 (10), 761-766.

(45) Deplace, F.; Carelli, C.; Mariot, S.; Retsos, H.; Chateauminois, A.; Ouzineb, K.; Creton, C. Fine Tuning the Adhesive Properties of a Soft Nanostructured Adhesive with Rheological Measurements. J. Adhes. 2009, 85 (1), 18-54. 
Table of contents graphic:

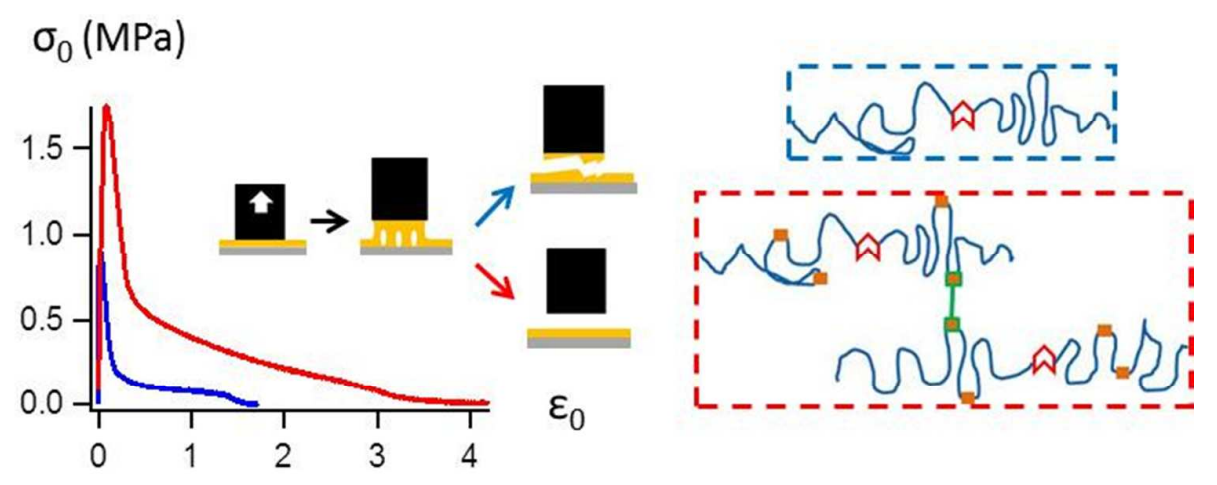

